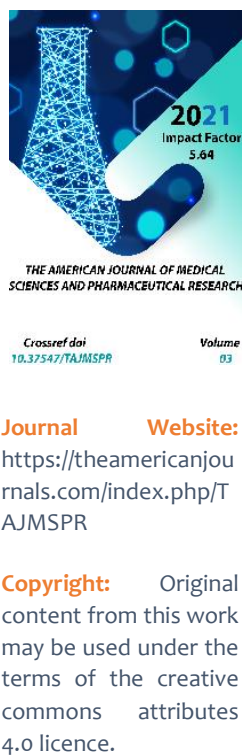

\section{The Country Of Dental Implanting, The Risks Involved}

\section{Shukurov Sherzod Shuxratovich}

Assistant Professor Of The Department Of Pediatric Dentistry Of Samarkand Medical Institute, Uzbekistan

Turaev Alimjan Baxriddinovich

Assistant Professor Of The Department Of Pediatric Dentistry Of Samarkand Medical Institute, Uzbekistan

\section{Arziqulova Munisa Shuxratovna}

Assistant Professor Of The Department Of Pediatric Dentistry Of Samarkand Medical Institute, Uzbekistan

Makhmudov Gulomjon Alisherovich

Assistant Professor Of The Department Of Pediatric Dentistry Of Samarkand Medical Institute, Uzbekistan

\title{
ABSTRACT
}

Success or failure of implants depends on the health of the person receiving the treatment, drugs which affect the chances of Osseo integration, and the health of the tissues in the mouth. The amount of stress that will be put on the implant and fixture during normal function is also evaluated. Planning the position and number of implants is key to the long-term health of the prosthetic since biomechanical forces created during chewing can be significant.

\section{KEYWORDS}

Dental Implanting, Jawbone, Procedure, Fake Teeth, Infection, Nerve Damage.

\section{INTRODUCTION}

A dental implant (also known as an end osseous implant or fixture) is a surgical component that interfaces with the bone of the jaw or skull to support a dental prosthesis such as a crown, bridge, denture, facial prosthesis or to act as an orthodontic anchor. The basis for modern dental implants is a biologic process called Osseo integration, in which materials such as titanium form an intimate bond to bone. The implant fixture is first placed so that it is likely to Osseo integrate, then a dental prosthetic is added. A variable amount of healing time is required for Osseo integration before either the dental prosthetic (a tooth, bridge or denture) is attached to the implant or an abutment is placed which will hold a dental prosthetic/crown. Dental implants are surgically placed in your jawbone, where they serve as the roots of missing teeth. Because the titanium in the implants fuses with your jawbone, the implants won't slip, make noise 
or cause bone damage the way fixed bridgework or dentures might. And the materials can't decay like your own teeth that support regular bridgework can.

In general, dental implants may be right for you if you:

Have one or more missing teeth;

Have a jawbone that's reached full growth;

Have adequate bone to secure the implants or are able to have a bone graft;

Have healthy oral tissues;

Don't have health conditions that will affect bone healing;

Are unable or unwilling to wear dentures;

Want to improve your speech;

Are willing to commit several months to the process;

Don't smoke tobacco.

The primary use of dental implants is to support dental prosthetics (i.e. false teeth). Modern dental implants make use of Osseo integration, the biologic process where bone fuses tightly to the surface of specific materials such as titanium and some ceramics. The integration of implant and bone can support physical loads for decades without failure.

The US has seen an increasing use of dental implants, with usage increasing from $0.7 \%$ of patients missing at least one tooth (1999 2000), to $5.7 \%$ (2015 - 2016), and was projected to potentially reach $26 \%$ in 2026 . Implants are used to replace missing individual teeth (single tooth restorations), multiple teeth, or to restore edentulous dental arches (implant retained fixed bridge, implant-supported over denture). Do note that alternative treatments to tooth loss are available (see Missing tooth replacement, Tooth Loss).

Historically, there have been two different types of dental implants:

End steal and sub periosteal.

End steal refers to an implant that is "in the bone," and sub periosteal refers to an implant that rests on top of the jawbone under the gum tissue. Sub periosteal implants are no longer in use today because of their poor long-term results in comparison to end steal dental implants.

\section{MATERIALS AND METHODS}

While the primary function of dental implants is for teeth replacement, there are areas in which implants can assist in other dental procedures. Due to their stability, dental implants can be used to support a removable denture and provide a more secure and comfortable fit. In addition, for orthodontics procedures, dental mini-implants can act as temporary anchorage devices (TAD) to help move teeth to a desired position. These miniimplants are small and temporarily fixed to bone while assisting in anchorage for teeth movement. They are subsequently removed after their function has been served.

During the consult and planning stage, the dental surgeon will visually examine the site in the mouth where a dental implant is being considered as well as look at dental imaging studies (X-rays, panoramic films, and/or CT scans). At this time, the quality and quantity of jawbone is assessed to determine if more bone is needed at the site. Once it has been established that a dental implant can be placed in the desired location, the patient will return for surgical procedures for the dental implant(s). During all surgical procedure 
appointments, the patient is usually given local anesthetic to numb the surgical area as well as any other sedatives necessary for comfort and anxiety.

The first stage of oral surgery often involves a tooth or teeth extraction. Oftentimes, the site of a dental implant still has an existing damaged tooth present. In order to prepare for placement of a dental implant, the tooth will need to be extracted. More often than not, an "alveolar bone graft" (cadaver or synthetic bone) is placed to achieve a solid base of bone for the implant. This site will be allowed to heal for two to six months. For a site that has no tooth and bone loss is present, it will require a different bone graft that is placed on top of existing jawbone ("online bone graft"). This procedure is more involved and usually requires about six or more months of healing. In some instances, when enough bone is present, the damaged tooth can be extracted followed by the implant placement procedure at the same appointment. This procedure is called "immediate implant" placement.

Dental implants are also used in orthodontics to provide anchorage (orthodontic mini implants). An evolving field is the use of implants to retain obturators (removable prosthesis used to fill a communication between the oral and maxillary or nasal cavities).Facial prosthetics, used to correct facial deformities (e.g. from cancer treatment or injuries) can use connections to implants placed in the facial bones. Depending on the situation the implant may be used to retain either a fixed or removable prosthetic that replaces part of the face.

Success or failure of implants depends on the health of the person receiving the treatment, drugs which affect the chances of Osseo integration, and the health of the tissues in the mouth. The amount of stress that will be put on the implant and fixture during normal function is also evaluated. Planning the position and number of implants is key to the long-term health of the prosthetic since biomechanical forces created during chewing can be significant. The position of implants is determined by the position and angle of adjacent teeth, by lab simulations or by using computed tomography with CAD/CAM simulations and surgical guides called stents. The prerequisites for long-term success of Osseo integrated dental implants are healthy bone and gingiva. Since both can atrophy after tooth extraction, pre-prosthetic procedures such as sinus lifts or gingival grafts are sometimes required to recreate ideal bone and gingiva.

The final prosthetic can be either fixed, where a person cannot remove the denture or teeth from their mouth, or removable, where they can remove the prosthetic. In each case an abutment is attached to the implant fixture. Where the prosthetic is fixed, the crown, bridge or denture is fixed to the abutment either with lag screws or with dental cement. Where the prosthetic is removable, a corresponding adapter is placed in the prosthetic so that the two pieces can be secured together.

The risks and complications related to implant therapy divide into those that occur during surgery (such as excessive bleeding or nerve injury), those that occur in the first six months (such as infection and failure to Osseo integrate) and those that occur long-term (such as peri-implantitis and mechanical failures). In the presence of healthy tissues, a well-integrated implant with appropriate biomechanical loads can have 5-year plus 
survival rates from 93 to 98 percent and 10 to 15 year lifespans for the prosthetic teeth. Longterm studies show a 16- to 20-year success (implants surviving without complications or revisions) between $52 \%$ and $76 \%$, with complications occurring up to $48 \%$ of the time. Risks like any surgery, dental implant surgery poses some health risks. Problems are rare, though, and when they do occur they're usually minor and easily treated. Risks include:

Infection at the implant site

Injury or damage to surrounding structures, such as other teeth or blood vessels

Nerve damage, which can cause pain, numbness or tingling in your natural teeth, gums, lips or chin

Sinus problems, when dental implants placed in the upper jaw protrude into one of your sinus cavities.

After the procedure

Whether you have dental implant surgery in one stage or multiple stages, you may experience some of the typical discomforts associated with any type of dental surgery, such as:

Swelling of your gums and face

Bruising of your skin and gums

Pain at the implant site

Minor bleeding

You may need pain medications or antibiotics after dental implant surgery. If swelling, discomfort or any other problem gets worse in the days after surgery, contact your oral surgeon.

After each stage of surgery, you may need to eat soft foods while the surgical site heals. Typically, your surgeon will use stitches that dissolve on their own. If your stitches aren't self-dissolving, your doctor removes them.

\section{RESULTS}

Most dental implants are successful. Sometimes, however, the bone fails to fuse sufficiently to the metal implant. Smoking, for example, may contribute to implant failure and complications. If the bone fails to fuse sufficiently, the implant is removed, the bone is cleaned up, and you can try the procedure again in about three months.

You can help your dental work - and remaining natural teeth - last longer if you:

\section{CONCLUSION}

Practice excellent oral hygiene. Just as with your natural teeth, keep implants, artificial teeth and gum tissue clean. Specially designed brushes, such as an interdental brush that slides between teeth, can help clean the nooks and crannies around teeth, gums and metal posts.

See your dentist regularly. Schedule dental checkups to ensure the health and proper functioning of your implants and follow the advice for professional cleanings.

Avoid damaging habits. Don't chew hard items, such as ice and hard candy, which can break your crowns - or your natural teeth. Avoid tooth-staining tobacco and caffeine products. Get treatment if you grind your teeth.

\section{REFERENCES}

1. A NEW STAGE OF REFORMING THE TRAINING OF LEGAL PERSONNEL. $F$ Mukhitdinova Review of law sciences 2 (1), 2017.

2. Ideas and teachings on the concept of spiritual and moral education of the younger generation in Uzbekistan FA 
The American Journal of Medical Sciences and Pharmaceutical Research

(ISSN - 2689-1026)

Published: September 30, 2021 | Pages: 142-146

IMPACT FACTOR

2021: 5.64

Doi: https://doi.org/10.37547/TAJMSPR/Volume03Issue09-23

Mukhitdinova, SS Agzamkhodzhayeva

Eurasian Union of Scientists, 2019.

3. Problems of power and management in the works of Eastern thinkers FA Mukhitdinova, HMM Mukhammedov High School, 35-38, 2015.

4. Implementation of human rights protection in Uzbekistan IV Kudryavtsev Eurasian Union of Scientists, 2019.

5. The origins of the idea of civil society FA Mukhitdinova, IN Kudryavtsev ACTUAL ASPECTS OF MODERN SCIENCE, 102-109, 2017. 